Acta Crystallographica Section B

Structural

Science

ISSN 0108-7681

Lukás Palatinus, Mongi Amamił and Sander van Smaalen*

Laboratory of Crystallography, University of Bayreuth, D-95440 Bayreuth, Germany

₹ Present address: Faculté de Sciénces de Sfax, Université de Sfax, Route de Soukra, 3018 Sfax, Tunisia.

Correspondence e-mail: smash@uni-bayreuth.de

\title{
Structure of incommensurate ammonium tetra- fluoroberyllate studied by structure refinements and the maximum entropy method
}

Incommensurately modulated ammonium tetrafluoroberyllate (AFB) occurs in a narrow temperature interval between the paraelectric room-temperature phase with space group Pnma $\left(T_{i}=178 \mathrm{~K}\right)$ and the ferroelectric low-temperature phase with space group $\operatorname{Pna}_{1}\left(T_{c}=173 \mathrm{~K}\right)$. The structure is determined from accurate single-crystal X-ray diffraction data collected with synchrotron radiation at $175 \mathrm{~K}$. The superspace group of the structure is Pnma( $\alpha 00) 0 s s$ with $\alpha=0.4796$ (4). Both structure refinements and the maximum entropy method lead to the same structure model, which involves only single harmonic modulations. The building units of the structure are $\mathrm{BeF}_{4}^{2-}$ and $\mathrm{NH}_{4}^{+}$complex ions with approximately tetrahedral point symmetry. They are relatively rigid and the modulations consist mainly of translations of the tetrahedra and their rotations around a fixed axis. The modulation is related to changes in the network of the hydrogen bonds. The lowtemperature superstructure can be described as a commensurately modulated structure with the same superspace symmetry. The first harmonic modulations of the lowtemperature and incommensurate phases are related by a scale factor with a value of approximately two. In addition, the low-temperature phase exhibits a second harmonic modulation that is responsible for shifts along $\mathbf{c}$ and the ferroelectricity in this phase. The experimental data of the incommensurate phase do not contain any evidence for the presence of a second harmonic in the modulation functions. This suggests that the development of the second harmonic, i.e. the development of the spontaneous polarization, is responsible for the lock-in transition.

\section{Introduction}

Ammonium tetrafluoroberyllate (AFB) is centrosymmetric with the space group Pnma at room temperature. It undergoes two phase transitions at lower temperatures. After the first transition at $T_{i}=182.9 \mathrm{~K}$ (Strukov et al., 1973; Makita \& Yamauchi, 1974) the structure becomes incommensurate with the modulation wavevector close to $0.5 \mathbf{a}^{*}$ (Iizumi \& Gesi, 1977). The second phase transition leads to a non-centrosymmetric ferroelectric phase (Pepinsky \& Jona, 1957). The reported values of $T_{c}$ vary between $174.2 \mathrm{~K}$ (Pepinsky \& Jona, 1957) and 177.2 K (Strukov et al., 1973). The crystal structures of the paraelectric and ferroelectric phases have been studied by X-ray diffraction (Onodera \& Shiozaki, 1979; Garg \& Srivastava, 1979) and neutron diffraction (Srivastava et al., 1999). O'Reilly et al. (1967) and Onodera \& Shiozaki (1979) suggest that one of the ammonium ions is orientationally
Received 3 July 2003

Accepted 12 January 2004
(C) 2004 International Union of Crystallography Printed in Great Britain - all rights reserved 
Table 1

Experimental data.

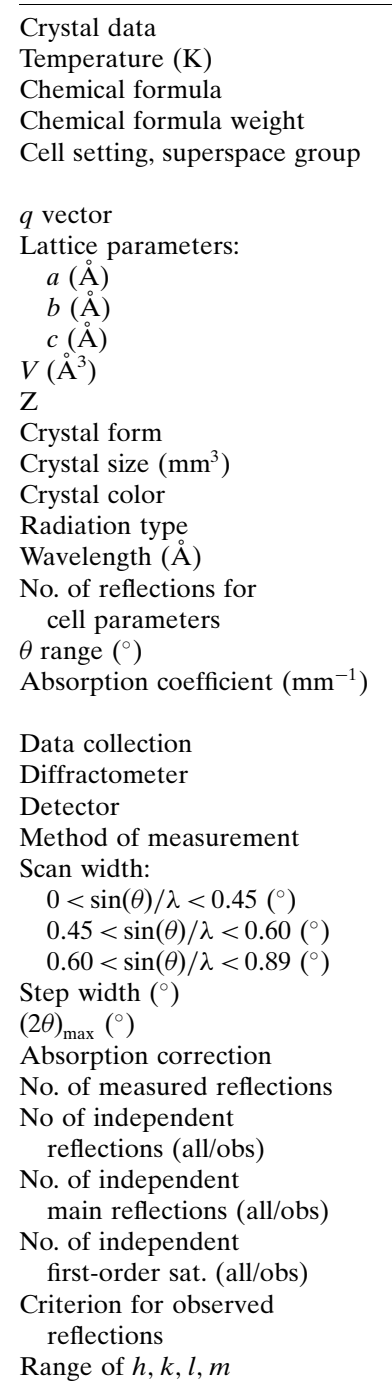

No. of standard reflections

Frequency of standard reflections

Refinement

Refinement on

Weighting scheme

$R_{\mathrm{obs}} / R_{\mathrm{all}} / w R_{\mathrm{obs}} / w R_{\mathrm{all}}$

All reflections

Main reflections

First-order satellites

Goodness-of-fit (obs/all)

No. of reflections

No. of parameters

$(\Delta / \rho)_{\max }$

$\Delta \rho_{\max }, \Delta \rho_{\min }\left(\mathrm{e} \AA^{-3}\right)$

Extinction correction

Extinction coefficient

Computer program used: JANA2000 (Petříček \& Dušek, 2000).

disordered in the paraelectric phase. O'Reilly et al. (1967) propose that the phase transition is of the order-disorder type. However, the structures by Garg \& Srivastava (1979) and the most recent work by Srivastava et al. (1999) do not indicate any disorder in the room-temperature structure. These authors conclude that the phase transition is a result of changes in the hydrogen-bonding scheme.

Iizumi \& Gesi (1977) have proposed a model of the phase transitions similar to that proposed for potassium selenate (Iizumi et al., 1977). In this model, the spontaneous polarization would already be present in the modulated phase on a microscopic scale, but the incommensurateness of the modulation would cause the average spontaneous polarization to be zero. The incommensurate-to-commensurate phase transition would then correspond to ordering of the directions of the microscopic polarizations, resulting in a macroscopic spontaneous polarization.

None of the previous works have studied the crystal structure of the intermediate incommensurate phase. Knowledge of the structure of this phase can give additional insight into the mechanism of the phase transitions in AFB and into the origin of the ferroelectricity of the low-temperature phase. In particular, we will show that the microscopic polarizations supposed by the model of Iizumi \& Gesi (1977) are insignificant in the incommensurate phase within the experimental resolution.

Single-crystal diffraction data were collected with synchrotron radiation. The structural model was found by refinements in superspace. The structure was further studied by means of the Maximum Entropy Method (MEM). The Maximum Entropy Method (MEM) is a general tool for a model-free reconstruction of positive additive distributions. One of many applications in crystallography is a reconstruction of the electron density from phased structure factors (Gilmore, 1996). The maximum entropy formalism can be extended to superspace (Steurer, 1991; van Smaalen et al., 2003). The MEM in superspace can give a non-parametric estimate of the shapes of the modulation functions. Thus, the MEM can be used to either independently confirm the refined model or to find a shape of modulation function that differs from the parametrized model (van Smaalen et al., 2003; Palatinus et al., 2004).

\section{Experimental}

Crystals of AFB were grown by slow evaporation at $278 \mathrm{~K}$ from an aqueous solution of a stoichiometric mixture of $\mathrm{NH}_{4} \mathrm{~F}$ (ACROS, ACS-Reagenz, purity $\geq 98 \%$ ) and $\mathrm{BeF}_{2}$ (Alfa Aesar, $99.9 \%$ purity of the metals basis). Several crystals were tested on a Nonius Mach3 diffractometer with a rotating anode generator and Mo $K \alpha$ radiation. A thick plate of the dimensions $0.20 \times 0.13 \times 0.08 \mathrm{~mm}^{3}$ was selected for the data collection. The diffraction data were collected on a Huber four-circle diffractometer at beamline D3 at Hasylab, DESY, Hamburg. The beam was monochromated by a $\operatorname{Si}(111)$ double-crystal monochromator. The wavelength was set to $0.7100 \AA$. An Oxford Cryojet was used to cool the sample by a cold nitrogen gas flow. Incommensurate satellite reflections were observed in the temperature range between 173 and $178 \mathrm{~K}$; the data were collected at $175 \mathrm{~K}$. Three standard 
reflections were measured every $2 \mathrm{~h}$ as a check of the stability of the experimental setup. Experimental details are given in Table 1.

The modulation wavevector was determined from the positions of 32 satellites. The value $q=0.4976$ (4) a* is very close to $\frac{1}{2}$. Thus, reflections of the type $h \mathrm{klm}$ are very close to

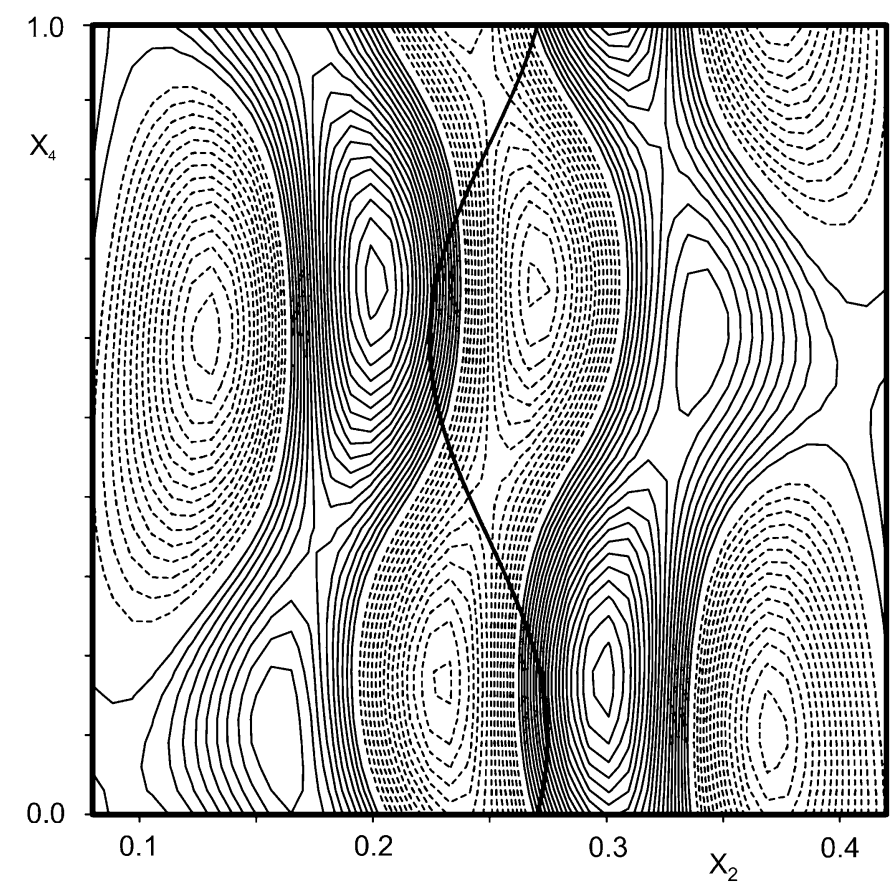

(a)

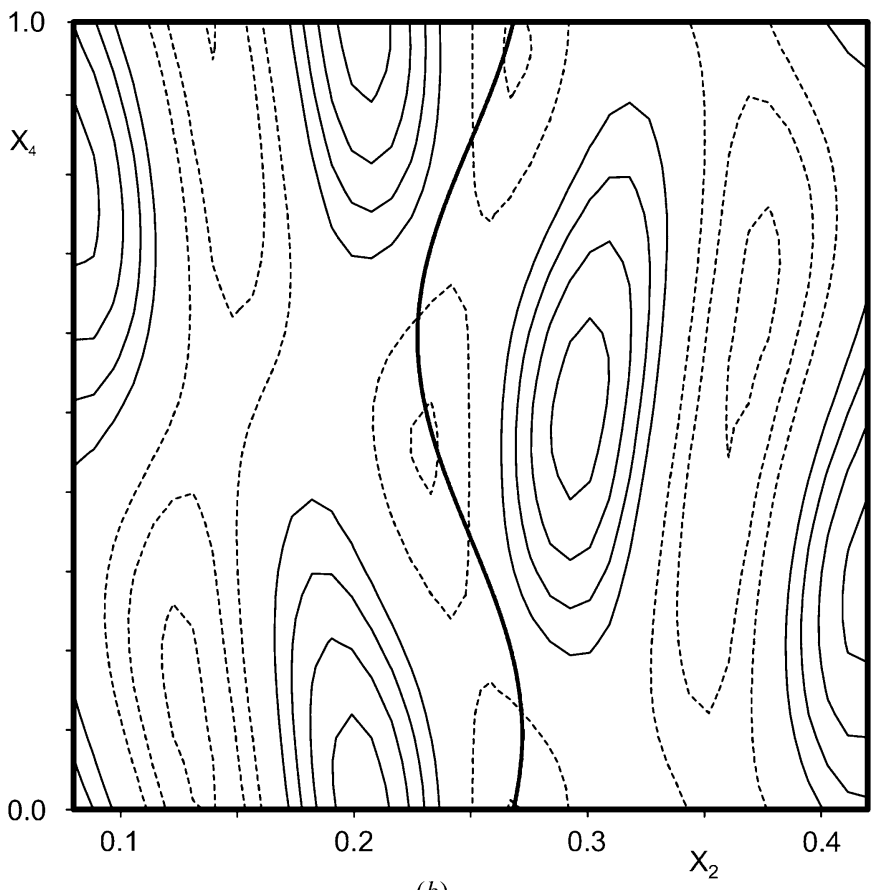

Figure 1

(b)

$x_{2}-x_{4}$ sections of the difference Fourier map at the position of the F2

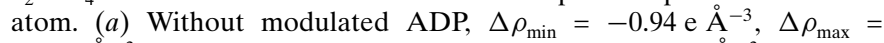
$0.90 \mathrm{e}^{-3}$. (b) With modulated ADP, $\Delta \rho_{\min }=-0.18 \mathrm{e}^{-3}, \Delta \rho_{\max }=$

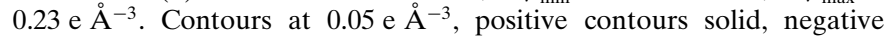
contours dashed, the zero contour omitted. The thick line corresponds to the refined position of the F2 atom. reflections $(h \pm 1) k l(m \mp 2)$. This can result in overlaps of these reflections in the $\omega$ scans. The value of the $\psi$ angle (rotation of the crystal around the scattering vector) was therefore optimized so that the distance between the positions of the reflections in the $\omega$ scans was maximal. Even with this procedure the occurrence of the two neighboring reflections in one scan could not always be avoided, but the distances between the peaks became large enough to allow intensities of the individual reflections to be determined.

The critical problem in the determination of the integrated intensities was the determination of the background. The standard procedures for background determination could not be used because of the presence of more than one peak in some scans. Therefore, the following procedure was used. Every profile was first smoothed by calculating a 13-point sliding average. Then a least-squares line was fitted to every 16 points of the smoothed profile. As a result, a smooth first derivative of the scan profile was obtained. Starting from the expected peak position, the derivative was scanned to the left. The first negative point, that was not followed by any significantly positive point, was considered to be the border of the peak area. On the right side, the procedure was repeated symmetrically. The peak area was determined for each peak in the profile and the points lying outside the peak areas were considered to be the background points. At least ten points were always assigned to the background at each side of the peak. The parameters of the method were selected empirically to produce the best results. In the case of the present data the method proved to be very robust and produced reliable estimates of the background.

The raw counts were corrected for the dead-time of the detector, and the integrated intensities were corrected for the Lp effects and for variation in the intensity of the primary beam. The absorption correction was not applied because of the very small absorption coefficient $\left(\mu=0.215 \mathrm{~mm}^{-1}\right)$.

The classes of reflections $(0 k l 0)$ with $k+l=2 n+1,(h k 0 m)$ with $h+m=2 n+1$ and $(h 0 l m)$ with $m=2 n+1$ were systematically extinct. No other extinction rule was observed. The unique superspace group corresponding to these extinction rules is Pnma $(\alpha 00) 0$ ss.

Second-order satellites $(m= \pm 2)$ were not observed in preliminary measurements. Therefore, these satellites were not measured during the data collection. However, due to the almost commensurate value of the $q$-vector the second-order satellites occur close to the main reflections. Analysis of the collected data set showed that profiles of 1131 main reflections (out of 1460) contained positions of the second-order satellites. All these 1131 profiles were visually checked and no peak was observed at the positions of the second-order satellite reflections.

\section{Structure refinements}

The structure was refined using the superspace approach (de Wolff et al., 1981; van Smaalen, 1995). All refinements were performed using the computer program JANA2000 (Petrríček $\&$ Dušek, 2000). The coordinates of the room-temperature 
Table 2

Basic structure coordinates of the incommensurate structure corresponding to the final refined model.

\begin{tabular}{llll}
\hline & $x$ & $y$ & $z$ \\
\hline Be & $0.25141(8)$ & $1 / 4$ & $0.41847(6)$ \\
F1 & $0.05442(9)$ & $1 / 4$ & $0.38015(8)$ \\
F2 & $0.27030(10)$ & $1 / 4$ & $0.56419(6)$ \\
F3 & $0.33820(7)$ & $0.03489(9)$ & $0.36575(5)$ \\
N1 & $0.18709(6)$ & $1 / 4$ & $0.09990(4)$ \\
N2 & $0.45778(6)$ & $1 / 4$ & $0.80326(4)$ \\
H11 & $0.3059(12)$ & $1 / 4$ & $0.1228(10)$ \\
H12 & $0.1075(16)$ & $1 / 4$ & $0.1586(12)$ \\
H13 & $0.1718(10)$ & $0.1298(15)$ & $0.0498(8)$ \\
H21 & $0.4876(13)$ & $1 / 4$ & $0.7213(10)$ \\
H22 & $0.5606(18)$ & $1 / 4$ & $0.8468(13)$ \\
H23 & $0.3946(12)$ & $0.1227(17)$ & $0.8194(8)$ \\
\hline
\end{tabular}

Table 3

Modulation functions of the displacive modulation.

For definitions see (1). Only first-order harmonic modulation functions were refined. Amplitudes are given in A.

\begin{tabular}{lllrrll}
\hline & $A_{x}^{1}$ & $B_{x}^{1}$ & \multicolumn{1}{c}{$A_{y}^{1}$} & \multicolumn{1}{c}{$B_{y}^{1}$} & $A_{z}^{1}$ & $B_{z}^{1}$ \\
\hline $\mathrm{Be}$ & 0 & 0 & $-0.0143(13)$ & $0.0085(12)$ & 0 & 0 \\
$\mathrm{~F} 1$ & 0 & 0 & $-0.0197(13)$ & $-0.0829(12)$ & 0 & 0 \\
$\mathrm{~F} 2$ & 0 & 0 & $0.0736(13)$ & $0.1095(12)$ & 0 & 0 \\
$\mathrm{~F} 3$ & $0.0251(9)$ & $0.0243(8)$ & $-0.0348(7)$ & $-0.0071(7)$ & $0.0828(8)$ & $0.0653(9)$ \\
$\mathrm{N} 1$ & 0 & 0 & $-0.0133(9)$ & $0.0254(9)$ & 0 & 0 \\
$\mathrm{~N} 2$ & 0 & 0 & $0.0338(9)$ & $-0.0067(9)$ & 0 & 0 \\
$\mathrm{H} 11$ & 0 & 0 & $-0.033(14)$ & $0.110(14)$ & 0 & 0 \\
$\mathrm{H} 12$ & 0 & 0 & $-0.123(17)$ & $0.040(16)$ & 0 & 0 \\
$\mathrm{H} 13$ & $-0.040(12)$ & $0.018(11)$ & $0.093(13)$ & $-0.065(13)$ & $-0.142(12)$ & $0.103(13)$ \\
$\mathrm{H} 21$ & 0 & 0 & $0.029(15)$ & $-0.020(13)$ & 0 & 0 \\
$\mathrm{H} 22$ & 0 & 0 & $0.003(19)$ & $-0.125(17)$ & 0 & 0 \\
$\mathrm{H} 23$ & $0.018(13)$ & $-0.128(13)$ & $0.017(14)$ & $0.061(14)$ & $0.004(11)$ & $-0.029(12)$ \\
\hline
\end{tabular}

structure (Srivastava et al., 1999) were used as a starting point for the refinement of the average structure. The positional parameters of all the atoms were refined together with the isotropic temperature parameters of $\mathrm{H}$ atoms and anisotropic harmonic temperature parameters of all the other atoms. The fit to main reflections converged to $R_{\text {main }}($ obs $)=0.043$. In the next step the modulation was introduced. The displacive modulations in the directions $i=1,2,3$ were described by the Fourier series

$$
u_{i}\left(\bar{x}_{s 4}\right)=\sum_{n=1}^{\infty} A_{i}^{n} \sin \left(2 \pi n \bar{x}_{s 4}\right)+B_{i}^{n} \cos \left(2 \pi n \bar{x}_{s 4}\right) .
$$

Functions with $n=1$ (first-order harmonic modulation) were refined for all atoms, starting from arbitrary but small values. The refinement with 97 parameters converged to $R_{\text {main }}(\mathrm{obs})=$ 0.042 and $R_{\text {sat }}(\mathrm{obs})=0.131$. The difference-Fourier maps showed significant structure around the positions of the $F$ atoms (Fig. 1a). The first harmonic modulation of the harmonic displacement parameters improved the fit only marginally.

It turned out that the fit can be significantly improved by introducing anharmonic displacement parameters (ADP) of the third order (Kuhs, 1992) for the F atoms as well as their first-order harmonic modulation. The $R$ values dropped to $R_{\text {main }}(\mathrm{obs})=0.028$ and $R_{\text {sat }}(\mathrm{obs})=0.066$ after the introduction of the modulated ADP. This decrease is significant, although the number of refinable parameters increased to 175 . The maxima in the difference electron density of all the $\mathrm{F}$ atoms decreased by a factor larger than three (Fig. $1 b$ ). There is no significant negative region of the probability density function at any of the three atoms. Moreover, the $\mathrm{BeF}_{4}$ tetrahedron becomes more regular in comparison with the refinement without ADPs. All these observations support the conclusion that the ADP parameters are the appropriate way to describe the structure. The positional parameters of the basic structure are listed in Table 2; the parameters of the modulation functions are listed in Table 3 . $^{\mathbf{1}}$

Another possibility to improve the fit is to introduce the second harmonic modulation of the harmonic displacement parameters. However, refinement of these parameters leads to higher $R$ values $\left[R_{\text {main }}(\mathrm{obs})=0.039\right.$, $R_{\text {sat }}($ obs $\left.)=0.076\right]$ than the refinement of the third-order ADPs and predicts unrealistically high intensities of the second-order satellites. The refinement of the second harmonic modulation is also very difficult without any observed second-order satellites. For these reasons this model was not adopted.

As mentioned in $\S 2$, we did not observe any second-order satellites. This indicates that the harmonic displacement modulation of second order is very weak or absent. Indeed, the introduction of the second-order harmonic displacement modulation did not improve the fit and did not lead to significant amplitudes of the second-order modulation functions.

The final structure model (first-order harmonic modulation only) produced calculated structure factors of the secondorder satellites that were small, but with some of them strong enough to be observable in our experiment. The intensities of the second-order satellites can be effectively decreased by introducing the second harmonic modulation of the temperature factors and refining this modulation against a data set, which contains the experimentally measured reflections together with the second-order satellites with zero intensity and small standard uncertainty. If the structural model is refined against this combined data set, the fit to the main reflections and first-order satellites remains essentially unchanged, while the calculated intensities of all the secondorder satellites become unobservable, in agreement with the experiment. This can be regarded as proof of the presence of some second harmonic component in the modulation of the

\footnotetext{
${ }^{\mathbf{1}}$ Supplementary data for this paper are available from the IUCr electronic archives (Reference: CK5001). Services for accessing these data are described at the back of the journal.
} 


\section{Table 4}

Basic structure coordinates of the superspace description of the lowtemperature structure, corresponding to the published coordinates of the structure at $163 \mathrm{~K}$ (Srivastava et al., 1999) with the positions of the $\mathrm{H}$ atoms corrected for differences between the neutron and X-ray positions (see text, §6).

The inaccuracies given in parentheses are combined inaccuracies of the average positions and modulation functions, because the inaccuracies of the separate parameters cannot be determined from the coordinates of the threedimensional model.

\begin{tabular}{llll}
\hline & $x$ & $y$ & $z$ \\
\hline Be & $0.2517(2)$ & $0.2500(3)$ & $0.4188(4)$ \\
F1 & $0.0560(3)$ & $0.2500(7)$ & $0.3769(6)$ \\
F2 & $0.2668(3)$ & $0.2500(6)$ & $0.5645(4)$ \\
F3 & $0.3397(3)$ & $0.0352(6)$ & $0.3666(5)$ \\
N1 & $0.1879(2)$ & $0.2500(3)$ & $0.1006(1)$ \\
N2 & $0.4559(2)$ & $0.2500(3)$ & $0.8029(3)$ \\
H11 & $0.3034(5)$ & $0.2500(16)$ & $0.1244(10)$ \\
H12 & $0.1124(7)$ & $0.2500(15)$ & $0.1665(11)$ \\
H13 & $0.1690(5)$ & $0.1278(12)$ & $0.0554(9)$ \\
H21 & $0.4816(6)$ & $0.2500(15)$ & $0.7188(8)$ \\
H22 & $0.5544(6)$ & $0.2500(15)$ & $0.8512(10)$ \\
H23 & $0.3930(6)$ & $0.1281(13)$ & $0.8206(8)$ \\
\hline
\end{tabular}

temperature factors, although the exact form of this modulation cannot be determined from the present data set, which does not contain any observed second-order satellites.

\section{The ferroelectric structure as a commensurately modulated structure}

The low-temperature phase can be described as a twofold superstructure of the room-temperature phase. Alternatively,

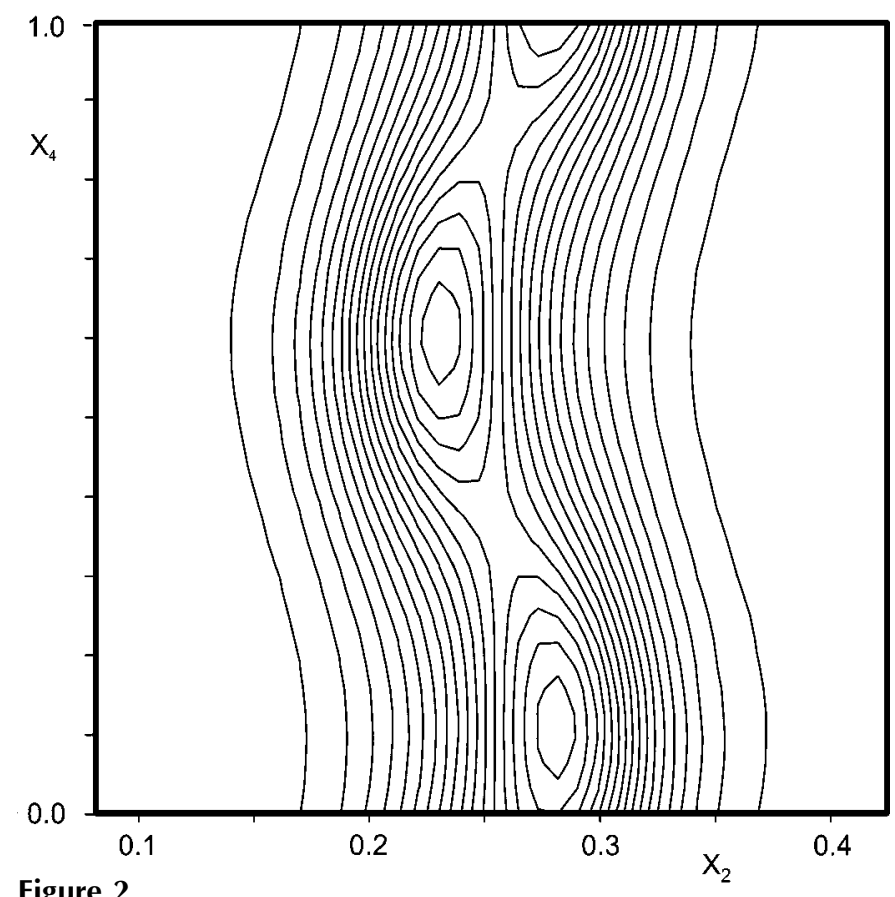

Figure 2

$x_{2}-x_{4}$ section of the MEM electron density at the position of the F2 atom (calculation obs 2 , Table 6). Contours at 2 e $\AA^{-3}$. The anharmonicity of the thermal displacement is observable as the asymmetric shape of the sections parallel to $x_{2}$. it can be described in superspace as a commensurately modulated structure with $q_{c}=0.5 \mathbf{a}^{*}$ and with the same superspace group as the incommensurate phase. For a given superspace group and modulation wavevector, the symmetry and structure of the supercell depend on the $t_{0}$ section of superspace (Yamamoto, 1982; van Smaalen, 1995). For Pnma $(\alpha 00) 0 s s$, Hogervorst (1986) has listed all the possible space groups of the supercell for different values of $t_{0}$ and different commensurate modulation wavevectors. The space group $P n a 2_{1}$ of the twofold supercell corresponds to the sections $t_{0}=1 / 8+n / 4, n=0,1,2,3$. Alternative values of $n$ correspond to a shift in the phase of the modulation or a shift in the origin of the three-dimensional space group. The actual value of $t_{0}$ for the transformation between the low-temperature structure reported by Srivastava et al. (1999) and the superspace model used in this work is $t_{0}=7 / 8$. Using this information, we have derived the modulation amplitudes of the superspace description for the low-temperature structure (Tables 4 and 5). Both first- and second-order harmonics were necessary to fit the supercell coordinates. Because of the commensurateness of the modulation $\left(q_{c}=0.5 \mathbf{a}^{*}\right)$, the phase and amplitude of the second-order harmonic are correlated.

$[\AA]$
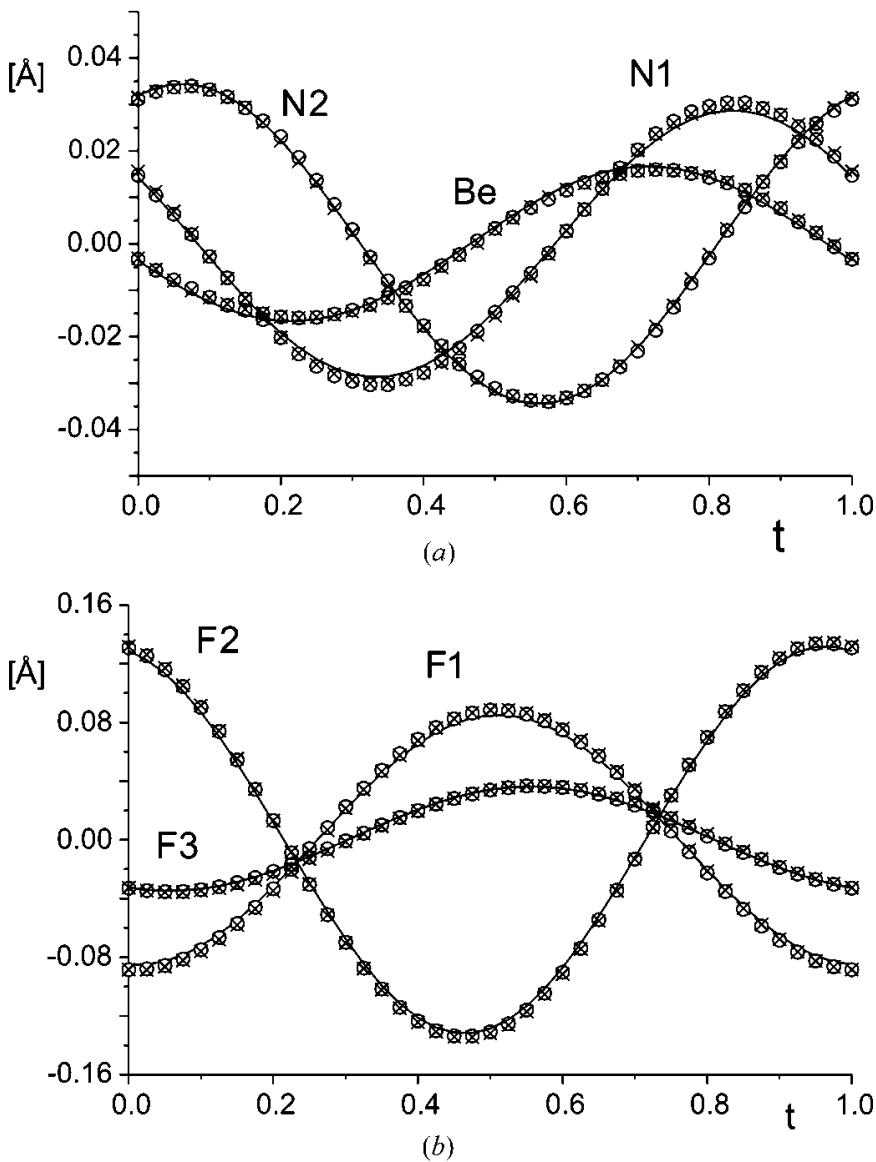

Figure 3

Modulation functions $u_{i}^{y}$ of the non-H atoms. Full lines: refined modulation functions; crosses: modulation functions derived from $\rho_{\mathrm{MEM}}$ of the calculation obs1; circles: modulation functions derived from $\rho_{\mathrm{MEM}}$ of the calculation obs2 (see Table 6). 


\section{Table 5}

Modulation functions of the superspace description of the low-temperature structure corresponding to the published coordinates of the structure at $163 \mathrm{~K}$ (Srivastava et al., 1999), with the positions of the $\mathrm{H}$ atoms corrected for differences between the neutron and X-ray positions (see text, §6).

For a definition of the symbols see (1). Amplitudes are given in $\AA$.

\begin{tabular}{lcccccc}
\hline & $A_{x}^{n}$ & $B_{x}^{n}$ & $A_{y}^{n}$ & $B_{y}^{n}$ & $A_{z}^{n}$ & $B_{z}^{n}$ \\
\hline$n=1$ & & & & & & \\
$\mathrm{Be}$ & 0 & 0 & -0.0311 & 0.0212 & 0 & 0 \\
$\mathrm{~F} 1$ & 0 & 0 & -0.0529 & -0.1810 & 0 & 0 \\
$\mathrm{~F} 2$ & 0 & 0 & 0.1669 & 0.2074 & 0 & 0 \\
$\mathrm{~F} 3$ & 0.0675 & 0.0485 & -0.0664 & 0.0018 & 0.1781 & 0.1125 \\
$\mathrm{~N} 1$ & 0 & 0 & -0.0270 & 0.0546 & 0 & 0 \\
$\mathrm{~N} 2$ & 0 & 0 & 0.0670 & -0.0047 & 0 & 0 \\
$\mathrm{H} 11$ & 0 & 0 & -0.0529 & 0.1974 & 0 & 0 \\
$\mathrm{H} 12$ & 0 & 0 & -0.2121 & 0.0993 & 0 & 0 \\
$\mathrm{H} 13$ & -0.0531 & 0.0478 & 0.1363 & -0.0570 & -0.2396 & 0.1625 \\
$\mathrm{H} 21$ & 0 & 0 & 0.0905 & 0.0000 & 0 & 0 \\
$\mathrm{H} 22$ & 0 & 0 & -0.0147 & 0.2180 & 0 & 0 \\
$\mathrm{H} 23$ & 0.0971 & -0.2177 & 0.0118 & 0.1351 & -0.0167 & -0.0313 \\
& & & & & & \\
$n=2$ & & & & & & \\
$\mathrm{Be}$ & 0.0000 & 0.0008 & 0 & 0 & 0.0000 & -0.0281 \\
$\mathrm{~F} 1$ & -0.0061 & 0.0023 & 0 & 0 & 0.0000 & 0.0000 \\
$\mathrm{~F} 2$ & 0.0000 & 0.0000 & 0 & 0 & -0.0031 & -0.0292 \\
$\mathrm{~F} 3$ & -0.0023 & -0.0030 & 0.0029 & 0.0047 & -0.0135 & -0.0219 \\
$\mathrm{~N} 1$ & 0.0008 & -0.0023 & 0 & 0 & 0.0063 & -0.0156 \\
$\mathrm{~N} 2$ & 0.0068 & 0.0015 & 0 & 0 & -0.0135 & -0.0042 \\
$\mathrm{H} 11$ & -0.0053 & -0.0144 & 0 & 0 & 0.0042 & 0.0115 \\
$\mathrm{H} 12$ & -0.0030 & 0.0023 & 0 & 0 & 0.0063 & -0.0052 \\
$\mathrm{H} 13$ & -0.0061 & 0.0106 & -0.0088 & 0.0153 & 0.0260 & -0.0458 \\
$\mathrm{H} 21$ & 0.0652 & 0.0076 & 0 & 0 & 0.0250 & 0.0031 \\
$\mathrm{H} 22$ & 0.0190 & -0.0068 & 0 & 0 & -0.0479 & 0.0167 \\
$\mathrm{H} 23$ & -0.0372 & -0.0296 & 0.0270 & 0.0212 & 0.0135 & 0.0104 \\
\hline
\end{tabular}

fied the positions of the $\mathrm{H}$ atoms in the low-temperature structure in such a way that the $\mathrm{N}-\mathrm{H}$ bonds are shorter than the published values by a factor of 0.9. This procedure is used only in Tables 4 and 5 and does not have any impact on the discussions about the hydrogen-bonding scheme in $\$ 6.2$.

\section{Maximum entropy method}

The superspace electron density of AFB was discretized on a grid of $128 \times 100 \times 162 \times 32$ pixels. This corresponds to a resolution of approximately $0.06 \AA$ in each realspace direction. The modulation functions were sampled at 32 points, allowing, in principle, the determination of up to eight harmonics. The experimental amplitudes of the structure factors, corrected for anomalous scattering and extinction, were combined with the phases of the best refined model to produce the input for the MEM calculation (Bagautdinov et al., 1998). This input is called 'observed data'. For checking We have chosen the phases so that the modulation amplitudes are minimal.

The average $\mathrm{N}-\mathrm{H}$ bond length in the low-temperature structure is $1.005 \AA$, while the presently determined incommensurate structure gives $0.900 \AA$. This difference is because of the different experimental techniques (neutron versus $\mathrm{X}$ ray scattering). In order to facilitate the comparison of the modulation amplitudes in the two structures, we have modi-

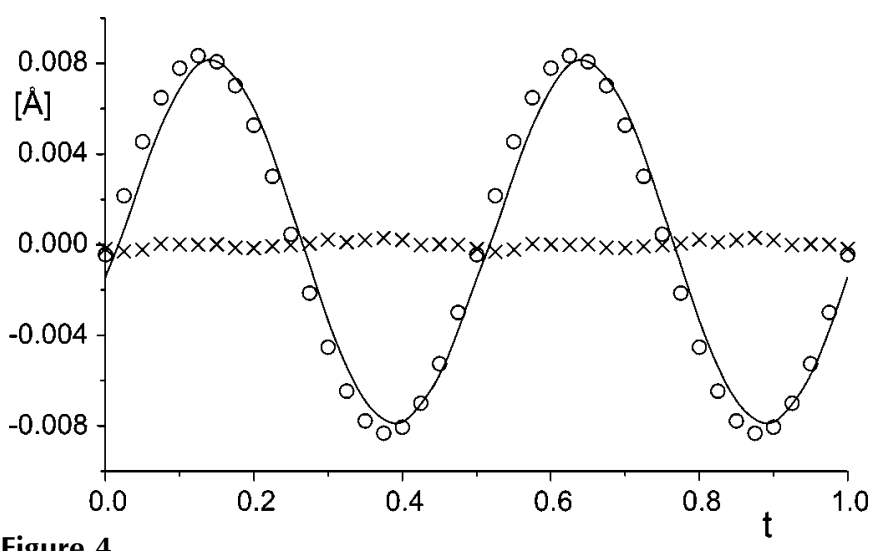

Figure 4

Modulation along $z$ of the $\mathrm{N} 1$ atom in the hypothetical incommensurate structure that includes second-order modulation functions (see §5). Full line: modulation derived from the coordinates of the ferroelectric structure. Circles: MEM reconstruction from simulated data including second-order satellites. Crosses: MEM reconstruction from simulated data with second-order satellites excluded from the input dataset. purposes, the MEM calculations were performed with the structure factors derived directly from the refined model ('calculated data'). The computer program BayMEM (van Smaalen et al., 2003) was used for two MaxEnt calculations, with different algorithms and different constraints for each data set (Table 6). Convergence was obtained for all four calculations.

The MaxEnt calculations result in optimized superspace electron densities $\rho_{\text {MEM }}$. $\rho_{\text {MEM }}$ exhibits local maxima in the form of strings parallel to the fourth dimension of superspace at the positions of the atoms (Fig. 2). The positions of the atoms as a function of the fourth coordinate have been determined by the computation of the centers of charge around each local maximum for different real-space sections (different values of $t$ ). The positions of the $\mathrm{H}$ atoms cannot be determined with this method, because these atoms do not form separate maxima in the electron density. The determination of the hydrogen positions and the investigation of the bonding electron density will be a topic of future research.

The agreement of the modulation functions derived from $\rho_{\text {MEM }}$ with the refined functions of the model is excellent (Fig. 3 ). The differences between the results by the MEM on the observed data and the refined functions are similar to or even smaller than the differences between the results by the MEM on calculated data and the refined functions. All differences in atomic positions are found to be below $10 \%$ of the pixel size. Thus, within the accuracy of the MEM calculations, the MEM shows perfect agreement with the refined model and the 
Table 6

Parameters of the MEM calculations.

S-S denotes the Sakata-Sato algorithm (Sakata \& Sato, 1990); Cambridge denotes the Cambridge algorithm (Skilling \& Bryan, 1948); F6 denotes the type of generalized F constraint (Palatinus \& van Smaalen, 2002); sw4 denotes the static weighting (De Vries et al., 1994) with weighting factor $w=1 /|\bar{H}|^{4}$, where $\bar{H}$ is the length of the diffraction vector.

\begin{tabular}{lllll}
\hline $\begin{array}{l}\text { Name of the } \\
\text { calculation }\end{array}$ & calc1 & calc2 & obs1 & obs2 \\
\hline $\begin{array}{l}\text { Pixel division } \\
\text { Pixel size }\left(\AA^{3}\right)\end{array}$ & $0.059 \times 0.059 \times 0.064$ & & & \\
$\begin{array}{l}\text { Input data } \\
\text { Algorithm }\end{array}$ & Calculated & Calculated & Observed & Observed \\
Constraint & F6 & Cambridge & $\mathrm{S}-\mathrm{S}$ & Cambridge \\
\hline
\end{tabular}

model is confirmed (van Smaalen et al., 2003). Inspection of $\rho_{\text {MEM }}$ also provides an independent indication of the modulated odd-order anharmonic temperature factors of the $\mathrm{F}$ atoms (Fig. 2).

A possible displacement along $\mathbf{c}$ of the atoms in special positions is of particular importance, because they are responsible for the spontaneous polarization in the lowtemperature phase. In the superspace approach the $z$ displacements are described by the second harmonics. Any evidence for the presence of second harmonics in the incommensurate structure has not been found in the MaxEnt calculations. We

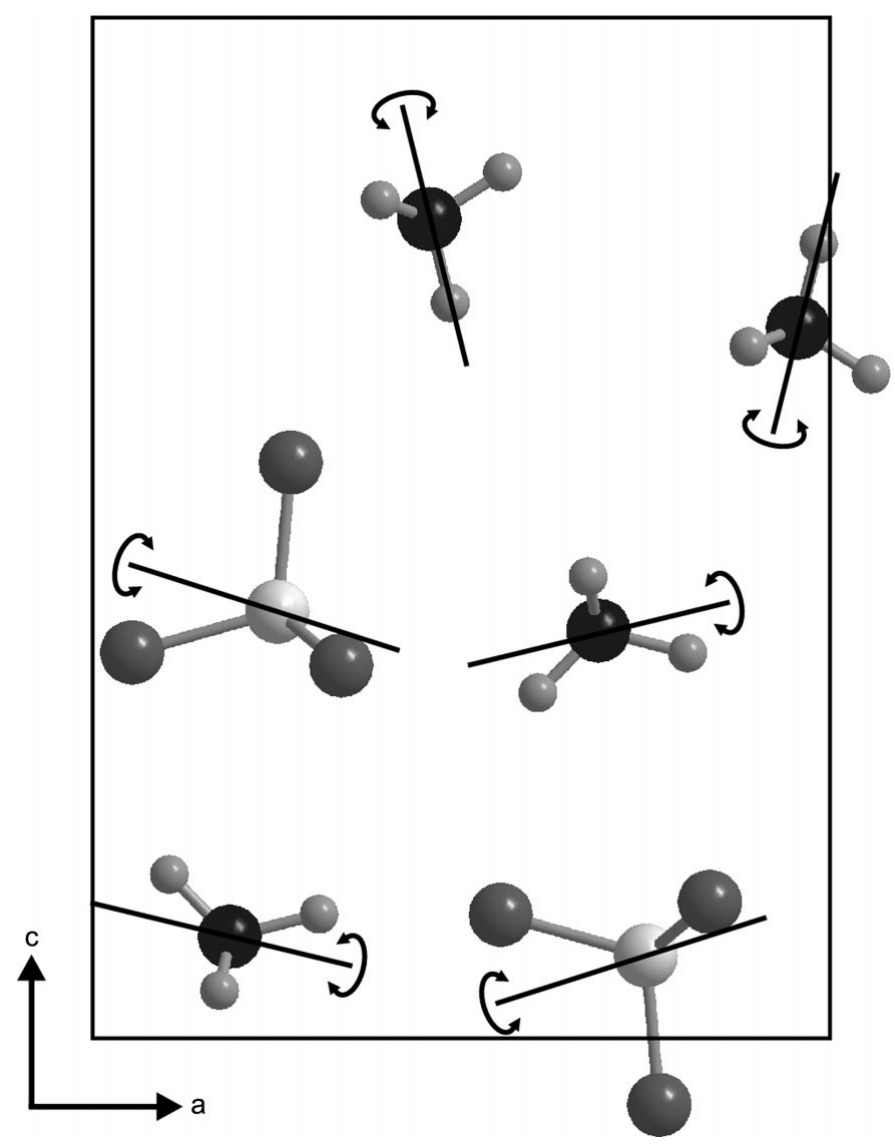

Figure 5

View of the basic structure of AFB along the $\mathbf{b}$ axis with axes of rotation of individual ions. Only ions with central atoms at $y=0.25$ are shown. Be atoms light gray, $\mathrm{N}$ atoms black. have tested the sensitivity of the MEM for second harmonic modulations by two additional calculations on simulated data. The structure model of the incommensurate phase (Table 3 ) is combined with second-order harmonic modulation functions, as they were obtained by dividing the second harmonics of the low-temperature structure by a factor of 2 (Table 5). The structure factors calculated from this model were used as input in the MEM calculations. The first calculation contained main reflections, and first- and second-order satellites; the second calculation only the main reflections and the first-order satellites. The results of the first calculation clearly reproduced the weak second-order modulations along $x$ and $z$. However, the results of the second calculation do not show any such modulations (Fig. 4). It can be concluded that it is not possible to detect the weak second-order modulations if the corresponding satellites have not been measured and included in the dataset, because the main reflections and first-order satellites do not contain enough information about this modulation.

\section{Discussion}

\subsection{Description of the modulated structure}

Among the seven structures of AFB published previously (see $\$ 1$ ), the work by Srivastava et al. (1999) is the most recent and it involves the most extensive data sets. The structures by Srivastava et al. (1999) basically confirm earlier refinements of the paraelectric and ferroelectric structures, but they are more accurate. Therefore, we compare the present structure of the incommensurate phase with the structures from Srivastava et al. (1999). If not otherwise stated, the expression 'RT structure' refers to the room-temperature structure, while the 'LT structure' indicates the twofold superstructure at $163 \mathrm{~K}$, as they were reported by Srivastava et al. (1999).

The $\mathrm{BeF}_{4}^{2-}$ complex anion has nearly perfect tetrahedral geometry. The small deviations from this symmetry are of equal sizes for the RT and incommensurate structures, while the LT structure exhibits slightly larger distortions (Table 7). The modulation has little effect on the geometry (Table 8). This implies that the modulation of the $\mathrm{BeF}_{4}^{2-}$ complex anion can be described as rigid-body translations and rotations in very good approximation.

A quantitative analysis shows that the modulation of $\mathrm{BeF}_{4}^{2-}$ can be described as the combined effect of small displacements along $\mathbf{b}$ and rotations around an axis in the mirror plane. While the angle of rotation varies with the phase of the modulation, the direction of this axis is fixed and is not affected by the modulation (Table 9, Fig. 5).

The deviations from tetrahedral symmetry are larger for the $\mathrm{NH}_{4}^{+}$cations than they are for the $\mathrm{BeF}_{4}^{2-}$ complex anions (Table 7). However, the variations of $0.04 \AA$ due to the modulations of the individual $\mathrm{N}-\mathrm{H}$ bond lengths (Table 8) are much smaller than the displacements up to $0.35 \AA$ of the $\mathrm{H}$ atoms due to the modulation. Similarly, the variations of the $\mathrm{H}-\mathrm{N}-\mathrm{H}$ angles due to the modulation (up to $4.6^{\circ}$ ) are much smaller than the rotations of the whole cations (Tables 8 and 
Table 7

Summary of the geometric parameters of the crystallographically independent complex ions in the incommensurate structure, roomtemperature structure and low-temperature structure.

The latter two structures are taken from Srivastava et al. (1999). $d_{\mathrm{av}}, d_{\min }$ and $d_{\max }$ are the average, minimal and maximal distance between the central atom and the ligand atoms of the complex ions, $\Delta_{d}=d_{\max }-d_{\min }$. $\alpha$ denotes the angles $\mathrm{F}-\mathrm{Be}-\mathrm{F}$ or $\mathrm{H}-\mathrm{N}-\mathrm{H}$

\begin{tabular}{|c|c|c|c|}
\hline & $\begin{array}{l}\text { Ferroelectric } \\
\text { phase }\end{array}$ & $\begin{array}{l}\text { Incommensurate } \\
\text { phase }\end{array}$ & $\begin{array}{l}\text { Paraelectric } \\
\text { phase }\end{array}$ \\
\hline Temperature (K) & 163 & 175 & 295 \\
\hline \multicolumn{4}{|l|}{$\mathrm{BeF}_{4}$} \\
\hline$d_{\mathrm{av}}$ & 1.535 & 1.5315 & 1.528 \\
\hline$d_{\min }$ & 1.524 & 1.5251 & 1.524 \\
\hline$d_{\max }$ & 1.550 & 1.5381 & 1.535 \\
\hline$\Delta_{d}$ & 0.026 & 0.0130 & 0.011 \\
\hline$\alpha_{\mathrm{av}}^{u}$ & 109.5 & 109.47 & 109.5 \\
\hline$\alpha_{\min }$ & 107.5 & 108.19 & 108.2 \\
\hline$\alpha_{\max }$ & 112.2 & 112.15 & 111.9 \\
\hline \multirow{2}{*}{\multicolumn{4}{|c|}{$\begin{array}{l}\Delta_{\alpha} \\
\mathrm{N}_{1} \mathrm{H}_{4}\end{array}$}} \\
\hline & & & \\
\hline$d_{\mathrm{av}}$ & 1.007 & 0.898 & 0.980 \\
\hline$d_{\min }$ & 0.989 & 0.857 & 0.964 \\
\hline$d_{\max }$ & 1.012 & 0.930 & 1.004 \\
\hline$\Delta_{d}$ & 0.044 & 0.073 & 0.040 \\
\hline$\alpha_{\mathrm{av}}^{a}$ & 109.5 & 109.3 & 109.5 \\
\hline$\alpha_{\min }$ & 105.2 & 104.2 & 107.3 \\
\hline$\alpha_{\max }$ & 115.4 & 119.5 & 115.4 \\
\hline$\Delta_{\alpha}$ & 10.21 & 5.3 & 8.1 \\
\hline \multicolumn{4}{|l|}{$\begin{array}{l}\stackrel{\Delta}{\alpha} \\
\mathrm{N} 2 \mathrm{H}_{4}\end{array}$} \\
\hline$d_{\mathrm{av}}$ & 1.003 & 0.901 & 0.991 \\
\hline$d_{\min }$ & 0.971 & 0.884 & 0.985 \\
\hline$d_{\max }$ & 1.030 & 0.926 & 1.000 \\
\hline$\Delta_{d}$ & 0.059 & 0.042 & 0.015 \\
\hline$\alpha_{\mathrm{av}}$ & 109.5 & 109.4 & 109.4 \\
\hline$\alpha_{\min }$ & 101.1 & 105.5 & 104.4 \\
\hline$\alpha_{\max }$ & 118.6 & 113.1 & 112.8 \\
\hline$\Delta_{\alpha}$ & 17.5 & 7.7 & 8.4 \\
\hline
\end{tabular}

9). The result is that the largest part of the modulations of the $\mathrm{NH}_{4}^{+}$ions is described by rigid-body modulations. The quantitative analysis again shows that the modulation is a combination of small displacements along $\mathbf{b}$ and rotations about a single axis in the mirror plane (Table 9, Fig. 5).

The modulation of the $\mathrm{NH}_{4}^{+}$cations affects the $\mathrm{N} 1-\mathrm{H} 13$ and $\mathrm{N} 2-\mathrm{H} 23$ bond lengths only, as well as a few bond angles involving either $\mathrm{H} 13$ or $\mathrm{H} 23$. The other bond lengths and angles are almost independent of the modulation (Table 8). The variation of deformations in the LT structure is slightly smaller for the $\mathrm{N}_{1} \mathrm{H}_{4}^{+}$cation, while it is much larger for the $\mathrm{N}_{2} \mathrm{H}_{4}^{+}$cation. For example, the $\mathrm{N} 2-\mathrm{H} 21$ bond length does not vary in the incommensurate structure, while it results in two distances different by $0.059 \AA$ in the LT structure. These differences are significant with respect to the standard uncertainties of the positions in both structures. We believe that the differences in distortions between the incommensurate and LT structures are related to the development of the spontaneous polarization in the latter phase.

The structure can be considered to consist of two alternating layers stacked along $\mathbf{c}$ (Fig. 6). Layer I is centered on $z$ $=0$, while layer II is centered on $z=0.5$. The translations and rotations of the complex ions within the same layer are correlated so that the neighboring ions have approximately the same deviations from an average position and orientation
Table 8

Selected interatomic distances $(\AA)$ and angles $\left(^{\circ}\right)$ in the refined structure of the incommensurate phase.

\begin{tabular}{lllll}
\hline & Average & Min & Max & $\Delta$ \\
\hline Be-F1 & $1.5370(9)$ & $1.5359(9)$ & $1.5381(9)$ & 0.002 \\
Be-F2 & $1.5302(9)$ & $1.5273(9)$ & $1.5331(9)$ & 0.006 \\
Be-F3 & $1.5294(14)$ & $1.5251(14)$ & $1.5334(14)$ & 0.008 \\
N1-H11 & $0.928(10)$ & $0.926(9)$ & $0.930(10)$ & 0.004 \\
N1-H12 & $0.860(12)$ & $0.857(9)$ & $0.864(12)$ & 0.007 \\
N1-H13 & $0.902(16)$ & $0.878(16)$ & $0.919(16)$ & 0.041 \\
N2-H21 & $0.884(11)$ & $0.884(11)$ & $0.884(11)$ & 0.000 \\
N2-H22 & $0.901(14)$ & $0.898(14)$ & $0.905(14)$ & 0.007 \\
N2-H23 & $0.909(16)$ & $0.895(17)$ & $0.926(16)$ & 0.031 \\
F1-Be-F2 & $110.52(5)$ & $110.43(5)$ & $110.61(5)$ & 0.2 \\
F1-Be-F3 & $108.55(8)$ & $108.19(8)$ & $108.81(8)$ & 0.6 \\
F2-Be-F3 & $108.58(8)$ & $108.46(8)$ & $108.73(8)$ & 0.3 \\
F3-Be-F3 & $112.06(6)$ & $111.98(6)$ & $112.15(6)$ & 0.2 \\
H11-N1-H12 & $118.9(11)$ & $118.4(11)$ & $119.5(10)$ & 1.1 \\
H11-N1-H13 & $106.2(12)$ & $104.2(12)$ & $108.1(12)$ & 3.9 \\
H12-N1-H13 & $109.4(14)$ & $107.0(14)$ & $111.6(14)$ & 4.6 \\
H13-N1-H13 & $105.7(14)$ & $105.5(14)$ & $105.8(14)$ & 0.3 \\
H21-N2-H22 & $105.6(11)$ & $105.5(11)$ & $105.7(11)$ & 0.2 \\
H21-N2-H23 & $108.2(13)$ & $107.6(12)$ & $108.8(13)$ & 1.2 \\
H22-N2-H23 & $111.1(14)$ & $109.1(14)$ & $113.1(14)$ & 4.1 \\
H23-N2-H23 & $112.3(14)$ & $112.1(14)$ & $112.5(24)$ & 0.4 \\
\hline
\end{tabular}

at the same value of $t$. The modulations of the ions of the second layer are shifted by approximately 0.25 in $t$. The result is such that at the places of the structure, where the ions of one

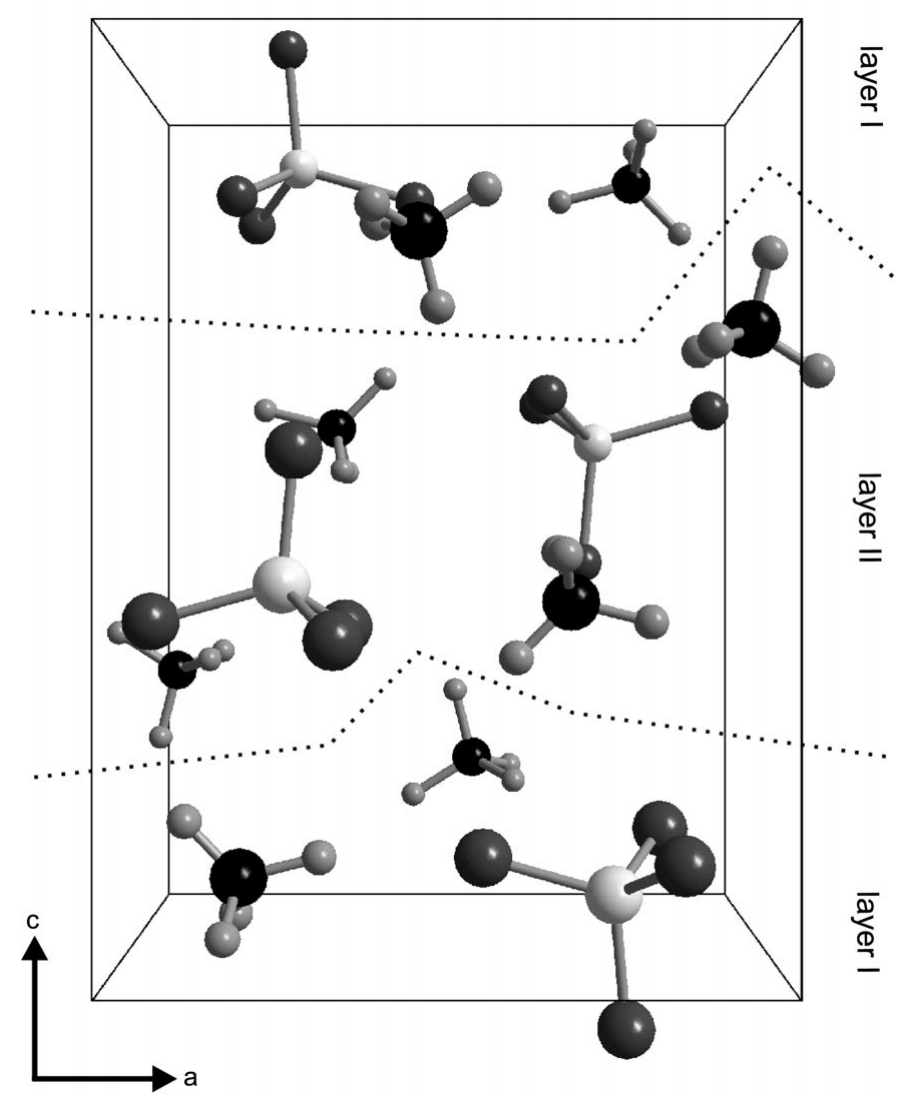

Figure 6

Perspective view of the basic structure along the $\mathbf{b}$ axis. Dotted lines separate the layers with correlated modulations of the ions (see $\S 6$ ). Be atoms are light gray, $\mathrm{N}$ atoms black. 
Table 9

Characteristics of the rotations of the complex ions $\left(^{\circ}\right)$.

The rotation axes lie in the mirror plane. The orientations of the axes are given as the oriented angle between the rotation axis and the positive a direction, with positive angles corresponding to the rotation towards the positive $\mathbf{c}$ axis. $\rho(A(i))$ is the angle that corresponds to the rotation of the atom $A(i)$ around the axis between the two most displaced positions. Atoms $A(i)$ correspond to atoms $\mathrm{F} i, \mathrm{H} 1 i$ and $\mathrm{H} 2 i$ for the $\mathrm{BeF}_{4}, \mathrm{~N}_{1} \mathrm{H}_{4}$ and $\mathrm{N}_{2} \mathrm{H}_{4}$ tetrahedra, respectively. The angle $\rho(\mathrm{H} 21)$ was not determined, because the atom $\mathrm{H} 21$ lies almost on the axis of rotation.

\begin{tabular}{|c|c|c|c|c|c|c|}
\hline & \multicolumn{2}{|l|}{$\mathrm{BeF}_{4}$} & \multicolumn{2}{|l|}{$\mathrm{N}_{1} \mathrm{H}_{4}$} & \multicolumn{2}{|l|}{$\mathrm{N} 2 \mathrm{H}_{4}$} \\
\hline & Inc. & LT & Inc. & LT & Inc. & LT \\
\hline Position of the axis & -17.7 & -20.9 & -13.6 & -13.5 & -76.1 & -82.8 \\
\hline$\rho(A(1))$ & 11.3 & 17.5 & 22.2 & 30.3 & - & - \\
\hline$\rho(A(2))$ & 10.2 & 15.2 & 15.0 & 28.9 & 14.8 & 24.5 \\
\hline$\rho(A(3))$ & 10.1 & 15.5 & 28.9 & 34.2 & 19.3 & 27.1 \\
\hline
\end{tabular}

these atoms. Note that the $\mathrm{H} 12$ atom rotates considerably less around the

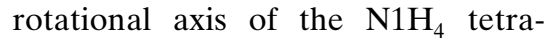
hedron than the other atoms and $\mathrm{H} 21$ lies almost exactly in the rotational axis of the $\mathrm{N}_{2} \mathrm{H}_{4}$ tetrahedron (Table 9).

The 'unstable' interactions involving the $\mathrm{H} 13$ and $\mathrm{H} 23$ atoms are subject to large changes of the $\mathrm{H} \cdots \mathrm{F}$ distance with the phase of the modulation. The result is that the $\mathrm{H} 13$ and $\mathrm{H} 23$ atoms of one $\mathrm{NH}_{4}^{+}$ion are alternatively bonded to $\mathrm{F} 2$ or $\mathrm{F} 3$ atoms of different $\mathrm{BeF}_{4}^{2-}$ anions,

layer reach their maximal deviations, the ions of the other layer are in their average positions and vice versa. The ions in layer I have the largest deviations approximately at $t=0.25$ and $t=0.75$; the ions in layer II are most displaced around $t=0.0$ and $t=0.5$. Because of the commensurateness of the LT structure, each ion adopts only four different orientations. The value $t_{0}=0.875$ corresponds to intermediate deviations of the ions in both layers.

As we will discuss below, the dissection of the structure into layers is strongly correlated with the modulations of the hydrogen bonds.

\subsection{Hydrogen-bonding scheme}

The interactions between the complex anions and cations are governed by ionic interactions and hydrogen bonds. Changes in the pattern of hydrogen bonds are believed to be responsible for the phase transitions in AFB (Onodera \& Shiozaki, 1979; Garg \& Srivastava, 1979; Srivastava et al., 1999).

The H.F distances as a function of $t$ are shown in Fig. 7. We take $2.6 \AA$ as the limit for the effective bonding interaction between $\mathrm{H}$ and $\mathrm{F}$ in the present structure. There is a gap between the distances below and above this limit. Inclusion of all the distances below this limit is necessary and sufficient to fully connect the structural units in a three-dimensional network of hydrogen bonds.

The H...F interactions can be roughly separated into two classes: 'stable' and 'unstable' interactions. The 'stable' interactions change only a little with the phase of the modulation. They involve the atoms H11, H12, H21 and H22, which lie in the mirror plane. H11 and H22 have only one very short distance to a neighboring $\mathrm{F}$ atom. This distance is not influenced by the modulation at all. These distances represent the strongest hydrogen-bond interactions in the structure and they remain almost unchanged in all three phases of AFB. The atoms $\mathrm{H} 12$ and $\mathrm{H} 21$ have three almost equally long distances to $\mathrm{F}$ atoms. These distances are considerably longer than the $\mathrm{H} 11 \cdots \mathrm{F}$ and $\mathrm{H} 22 \cdots \mathrm{F}$ distances and they can be considered weak, but the simultaneous occurrence of three such interactions in different directions stabilizes the position of the $\mathrm{H}$ atoms and is probably responsible for the special behavior of depending on the phase of the modulation. The formation of one very short and one very long $\mathrm{H} \cdots \mathrm{F}$ distance for each of the $\mathrm{H} 13$ and $\mathrm{H} 23$ atoms will have lower energy than all distances being of intermediate length, as found in the RT structure. The modulation of these bonds thus appears to be the driving force for the phase transition.

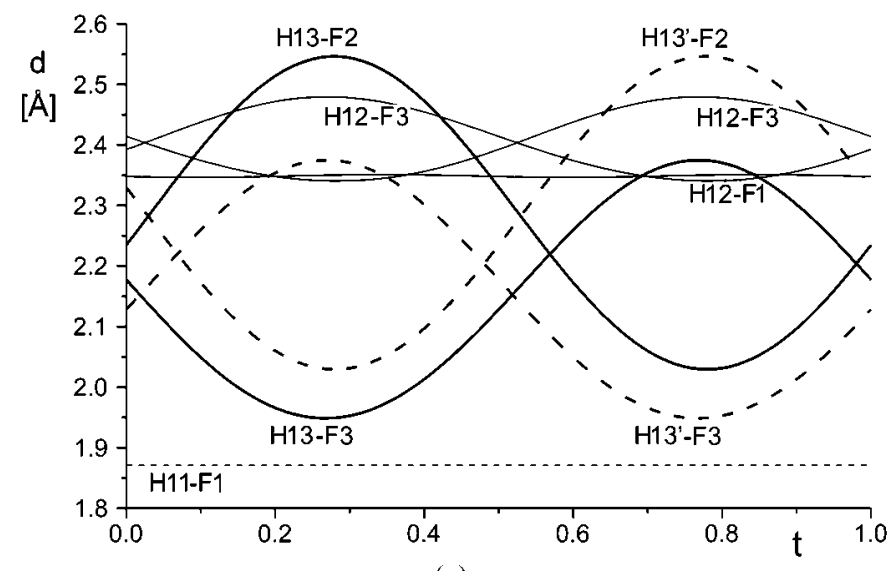

(a)

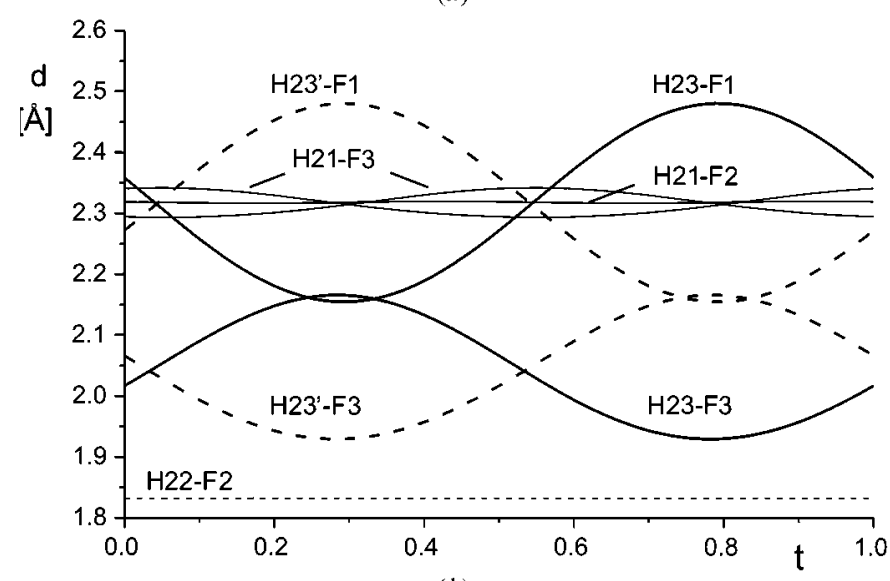

Figure 7

(b)

$\mathrm{H}$. . F distances shorter than $2.6 \AA$ as a function of $t$. (a) H atoms of the $\mathrm{N}_{1} \mathrm{H}_{4}^{+}$ion; (b) $\mathrm{H}$ atoms of the $\mathrm{N}_{2} \mathrm{H}_{4}^{+}$ion. All $\mathrm{H}$ atoms belong to layer $\mathrm{I}$. The primed $\mathrm{H}$ atoms have the symmetry code $x_{1}, \frac{1}{2}-x_{2}, x_{3}, \frac{1}{2}+x_{4}$. Curves of the same type represent distances to one $\mathrm{H}$ atom. Each curve represents the distance to a different $\mathrm{F}$ atom. The symmetry codes of the $\mathrm{F}$ atoms are omitted. 
All the inter-layer hydrogen bonds belong to the 'stable' interactions. The changes in the hydrogen-bonding scheme occur only within the layers and the bonds between the layers are not influenced by the modulation.

The infinite number of different $\mathrm{H}$..F distances described by each curve in Fig. 7 reduces to four different distances in the LT structure. In each 'unstable' interaction, two of the four distances represent the stronger $\mathrm{H}$. . F interaction and two the weak or broken interaction. However, no general distance limit can be defined that separates the stronger and weaker interactions within the modulated distances. The limit $2.1 \AA$ used by Srivastava et al. (1999) yields 13 strong hydrogen bonds involving four crystallographically independent ammonium ions, but these bonds involve both stronger and weaker $\mathrm{H} \cdots \mathrm{F}$ bonds on the $\mathrm{H} 23 \cdots \mathrm{F} 3$ curve, and neglect one of the shorter distances on the H23 ․ F1 curve. Obviously, it is inappropriate to reduce the description of the hydrogen-bond interactions in the LT structure of AFB to a simple categorization of the bonds to strong and weak based only on the distance limit.

\subsection{The ferroelectric phase transition}

The superspace description reveals a striking similarity between the incommensurate and the LT structures. The phases of the first harmonic modulation functions in the two structures are almost equal. The amplitudes of these modulation functions in the LT structure are approximately two times larger (Tables 3 and 5). The LT structure also contains second-order harmonic displacements that are responsible for the ferroelectricity, as is demonstrated by the following argument: All central atoms of the complex ions lie on special positions in superspace mirror planes. Symmetry restricts the first-order harmonic modulations of these atoms to shifts along the $y$ axis. The vectors representing the dipole moments of the $\mathrm{NH}_{4}^{+}$and $\mathrm{BeF}_{4}^{2-}$ complex ions also lie in the mirror planes and they are subject to the same symmetry restrictions. As a consequence, the first-order modulation cannot change the $z$ components of the dipole moments of the individual complex ions, nor can it create a $z$ component of the dipole moment by relative shifts of the $\mathrm{NH}_{4}^{+}$and $\mathrm{BeF}_{4}^{2-}$ ions. However, the small spontaneous polarization in the LT structure is along c, because this is the polar axis of the space group of the LT structure. Consequently, second-order harmonic modulations, which include displacements along a and $\mathbf{c}$, are necessary to describe the spontaneous polarization.

Second-order harmonic modulation amplitudes have not been found in the incommensurate structure, although neither the MEM nor the refinements could disprove the possibility of small second-order amplitudes. To analyze this further we have performed a series of calculations to estimate the upper boundary on the amplitude of the second harmonic that is consistent with our experiment. The following points of concern were raised: (i) The experiment did not reveal any observable intensity of the second-order satellites. Therefore, any modulation that leads to observable intensities of the calculated second-order satellites is inconsistent with the experiment.

(ii) The second harmonic modulation of the temperature factors is present in the structure (see $\$ 3$ ), but it is impossible to find its exact parameters. To obtain the upper boundary on the amplitudes of the second harmonic positional modulation, the temperature modulation has to be refined so as to lower the intensity of the satellites as much as possible.

The experimentally measured reflections were analyzed to obtain an estimate of the limit of observability. A fraction of $95 \%$ of the 2291 unobserved reflections $[I<3 \sigma(I)]$ have an intensity lower than $I_{\text {limit }}=0.412\left(I=|F|^{2}\right.$ with $F$ scaled to correspond to Fourier transform of one unit cell). Therefore, this value was taken as the lowest intensity that could have been observed in the experiment. The standard uncertainties of the second-order satellites were estimated as 0.09 on the absolute scale, which corresponds to the lower limit of the experimental standard uncertainties of all reflections. The results are not influenced by the exact value of the standard uncertainties.

Several hypothetical structure models were created by combining the final structure model with the second harmonic modulation derived from the LT structure with amplitudes multiplied by a constant factor $f_{2 \text { nd }}$. Each model was refined against the experimental data set combined with the secondorder satellites with zero intensity and a standard uncertainty equal to 0.09 . All parameters of the model were refined; only the second harmonic positional modulation was kept constant. After the refinement the intensities of the second-order satellites corresponding to the refined model were calculated. The number of observed intensities of the second-order satellites was $0,0,0,2,8,17$ for $f_{2 \text { nd }}=0.1,0.2,0.3,0.4,0.5,0.6$, respectively. The lowest $f_{2 \text { nd }}$ that leads to observable intensities is 0.4 of the original amplitudes (Table 5). Because the procedure described above was designed to suppress the intensities as much as possible, the occurrence of observable intensities indicates that this amount of modulation is inconsistent with the experiment. Thus, the amplitudes of the second-order harmonic positional modulation in the modulated structure are smaller than 0.4 times the amplitudes derived from the LT structure.

Any spontaneous polarization of the incommensurate phase is forbidden by the centrosymmetric superspace group, although a local dipole moment of the basic structure unit cell would be possible if a second-order harmonic displacement modulation is present. Because we did not obtain any evidence for such a second-order harmonic, our experiment does not support the model proposed by Iizumi et al. (1977) for the phase transition in potassium selenate and applied to AFB by Iizumi \& Gesi (1977). These authors propose that the incommensurate phase contains a modulated local dipole moment that, at the lock-in transition, orders to form the spontaneous polarization in the commensurate phase. Our analysis of the incommensurate structure indicates that the local dipole moments are very small and might even be absent 
in the modulated structure. This suggests that the dipole moment responsible for the observed spontaneous polarization might be formed at the lock-in transition.

If this conclusion is considered and if it is further considered that the first harmonics are sufficient to stabilize the pattern of hydrogen bonds (see §6.2), we obtain the following possibilities for the mechanisms of the phase transitions: The transition at $T_{i}$ is most probably governed by changes in the hydrogen bonding, in accordance with previous suggestions (Onodera \& Shiozaki, 1979; Garg \& Srivastava, 1979; Srivastava et al., 1999). The formation of the spontaneous polarization (forbidden in the incommensurate structure) might be the driving force for the transition at $T_{c}$. Alternatively, the rearrangements of the hydrogen bonds might also be responsible for the lock-in transition at $T_{c}$ and the spontaneous polarization would be a side effect of this rearrangement. However, the absence of significant local dipole moments in the incommensurate structure and the similarity of the overall pattern of the hydrogen-bonding scheme in the incommensurate and LT structures suggest that the spontaneous polarization is important for the stabilization of the LT lock-in phase, thus making the first mechanism the most probable one.

\section{Conclusions}

The atomic structure of the incommensurately modulated phase of ammonium tetrafluoroberyllate has been determined at $T=175 \mathrm{~K}$. The transition from the paraelectric phase to the incommensurate phase is found to be due to rearrangements in the hydrogen-bonding scheme. The structure of AFB can be described as an alternate stacking of two layers along $\mathbf{c}$. In the incommensurate structure $\mathrm{H} \cdots \mathrm{F}$ distances between the layers remain constant at their values in the RT phase. Within the layers, some of the $\mathrm{H} \cdots \mathrm{F}$ distances strongly vary between values corresponding to strong and very weak hydrogen bonds. This change is the driving force for the phase transition at $T_{i}$.

A microscopic polarization is found to be very small or absent in the modulated structure. The presence of this microscopic polarization in the incommensurate structure is supposed in the mechanism proposed by Iizumi \& Gesi (1977). At $T_{c}$ the local polarization would rearrange to form the LT structure with a spontaneous polarization. Instead, we do not find evidence for a local polarization in the incommensurate structure within the sensitivity of our experiment. Further specialized experiments are necessary that will determine the amount of the local polarization in the incommensurate structure with higher precision and possibly support the hypothesis of zero local polarization.

Two mechanisms can be envisaged for the transition at $T_{c}$. The first is further rearrangements in the hydrogen-bonding scheme, with the spontaneous polarization as an 'accidental' corollary. The second, more likely mechanism is that the development of the spontaneous polarization is the driving force for the lock-in transition.

Interesting questions pertaining to the mechanisms of the transitions remain. For example, it could be possible that the second-order harmonic modulation (and consequently local dipole moments) develops in the incommensurate phase close to $T_{c}$ or that the second-order harmonics develop as critical fluctuations. Whether this is true or not can be investigated by high-resolution diffraction experiments measuring the temperature dependence of the second-order satellites, as is possible at the third-generation synchrotron sources.

The authors thank K.-L. Stork (Laboratory of Crystallography, University of Bayreuth) for growing the crystals, and P. Daniels and W. Morgenroth for assistance with the synchrotron experiment. The financial support by the Deutsche Forschungsgemeinschaft (DFG) is gratefully acknowledged. The X-ray diffraction experiments were performed at beamline D3 of Hasylab at DESY.

\section{References}

Bagautdinov, B., Luedecke, J., Schneider, M. \& van Smaalen, S. (1998). Acta Cryst. B54, 626-634.

Becker, P. J. \& Coppens, P. (1974). Acta Cryst. A30, 129-152.

De Vries, R. Y., Briels, W. J. \& Feil, D. (1994). Acta Cryst. A50, $383-$ 391.

Garg, A. \& Srivastava, R. C. (1979). Acta Cryst. B35, 1429-1432.

Gilmore, C. J. (1996). Acta Cryst. A52, 561-589.

Hogervorst, A. C. R. (1986). PhD thesis. Delft, The Netherlands. Iizumi, M., Axe, J. D., Shirane, G. \& Shimaoka, K. (1977). Phys. Rev. $B, \mathbf{1 5}, 4392-4411$.

Iizumi \& Gesi, I. M. (1977). Solid State Commun. 22, 37-39.

Kuhs, W. F. (1992). Acta Cryst. A48, 80-98.

Makita, Y. \& Yamauchi, Y. (1974). J. Phys. Soc. Jpn, 37, 1470-1470.

Onodera, A. \& Shiozaki, Y. (1979). J. Phys. Soc. Jpn, 46, 157-166.

O'Reilly, D. E., Peterson, E. M. \& Tsang, T. (1967). Phys. Rev. 160, 333-336.

Palatinus, L. \& van Smaalen, S. (2002). Acta Cryst. A58, 559-567.

Palatinus, L., van Smaalen, S., McMahon, M. I., Degtyareva, O. \& Nelmes, R. J. (2004). In preparation.

Pepinsky, R. \& Jona, F. (1957). Phys. Rev. 105, 344-347.

Petrríček, V. \& Dušek, M. (2000). JANA2000. Institute of Physics, Praha, Czech Republic.

Sakata, M. \& Sato, M. (1990). Acta Cryst. A46, 263-270.

Skilling, J. \& Bryan, R. K. (1984). Mon. Not. R. Astr. Soc. 211, 111124.

Smaalen, S. van (1995). Crystallogr. Rev. 4, 79-202.

Smaalen, S. van, Palatinus, L. \& Schneider, M. (2003). Acta Cryst. A59, 459-469.

Srivastava, R. C., Klooster, W. T. \& Koetzle, T. F. (1999). Acta Cryst. B55, 17-23.

Steurer, W. (1991). Methods of Structural Analysis of Modulated Structures and Quasicrystals, edited by J. M. Perez-Mato, F. J. Zuniga \& G. Madariaga, pp. 344-349. Singapore: World Scientific.

Strukov, B. A., Skomorokhova, T. L., Koptsik, V. A., Boiko, A. A. \& Izrailenko, A. N. (1973). Sov. Phys. Cryst. 18, 86-88.

Wolff, P. M. de, Janssen, T. \& Janner, A. (1981). Acta Cryst. A37, 625636.

Yamamoto, A. (1982). Acta Cryst. A38, 87-92. 